\title{
The cholera spread Simulation
}

\author{
$N$ Baturina $^{1,}$, and $G$ Anisimova ${ }^{1,2}$ \\ ${ }^{1}$ Don State Technical University, 1, Gagarina sq., Rostov-on-Don, 344000, Russia \\ ${ }^{2}$ FKUZ "Rostov-on-Don Research Anti-Plague Institute"
}

\begin{abstract}
One of the frequently used modern epidemiological methods is the Simulation of disease spread. We used AnyLogic simulation. During the model construction, we take into account the specific cholera features. They are: the pathways of infection transmission, the course duration and the duration of the incubation (latent) period, the possibility of vaccination, etc. Two approaches are presented here: system-dynamic model and agentbased one. The system-dynamic model is used for strategic modelling of the epidemiological situation, it reflects the global trends. The agent-based approach allows describing the individual behaviour of each agent - person, who independently form events conditioning transitions between states.Used together they make it possible to reflect different aspects of the epidemiological process development. The combination of these two models gives more possibilities for their application in a real situation: give the opportunity to impose tactical nuances on strategic modelling.
\end{abstract}

\section{Introduction}

Research epidemiological methods are quite diverse [1, 2]. They are classic methods, such as statistical analysis, like trend and forecast [3,4], as well as modern methods, like simulation and so on. One can choose the research method in dependence of the research objectives and the special disease peculiarities. Usually, by using different approaches a more detailed analysis can be carried out [5].

One of the frequently used method is the Simulation of disease spread. There are many different methods of building a model. In many ways, the choice of the concrete methods of building a Simulation model is determined by a specific disease.

The specific features depending on the disease, which must be taken into account when constructing the model, includes the pathways of infection transmission, the course duration and the duration of the incubation (latent) period, the possibility of vaccination, etc.

In addition, it is necessary to take into account the specifics of the territory: both physicalgeographical and climatic features, as well as social, cultural characteristics, including the population density and closeness of communication between the people, the traditions of the region and so on.

The ways of transmission of cholera infection by contact, water and food were taken into account to build this model of cholera spread. It was taken into account the influence of

\footnotetext{
*Corresponding author: baturinata@mail.ru
} 
importation on the spread of infection, as well as a decrease in the rate of spread due to vaccination.

We considered two approaches: system-dynamic and agent-based one. Used together they make it possible to reflect different aspects of the epidemiological process development. It was used AnyLogic simulation.

The system-dynamic model assumes a high level of aggregation of objects. Here individuals are not distinguishable, the peculiarities of their behavior are not taken into account, and therefore this model is more intended for strategic modeling of the epidemiological situation. It may reflect global trends, for example, one of the most important in the spread of cholera feature - the flows of infection importation.

In contrast to the system dynamics model, the agent-based model is individual-oriented. It allows taking into account the behavior and psychology of people (attitudes towards travel, vaccinations during an epidemic, preventive measures to reduce the risks of infection).

\section{Stochastic model of the epidemic spread as a model of system dynamics}

The developed model of system dynamics is an extension of the classic SEIR model [6], which takes into account the ways of infection (contact, water and food), as well as imports.

Figure 1 shows a diagram of the levels and flows of the model, in Table 1 - their description, as well as a description of the parameters and variables used in the diagram. The model parameters were chosen both fixed and random with given distribution laws.

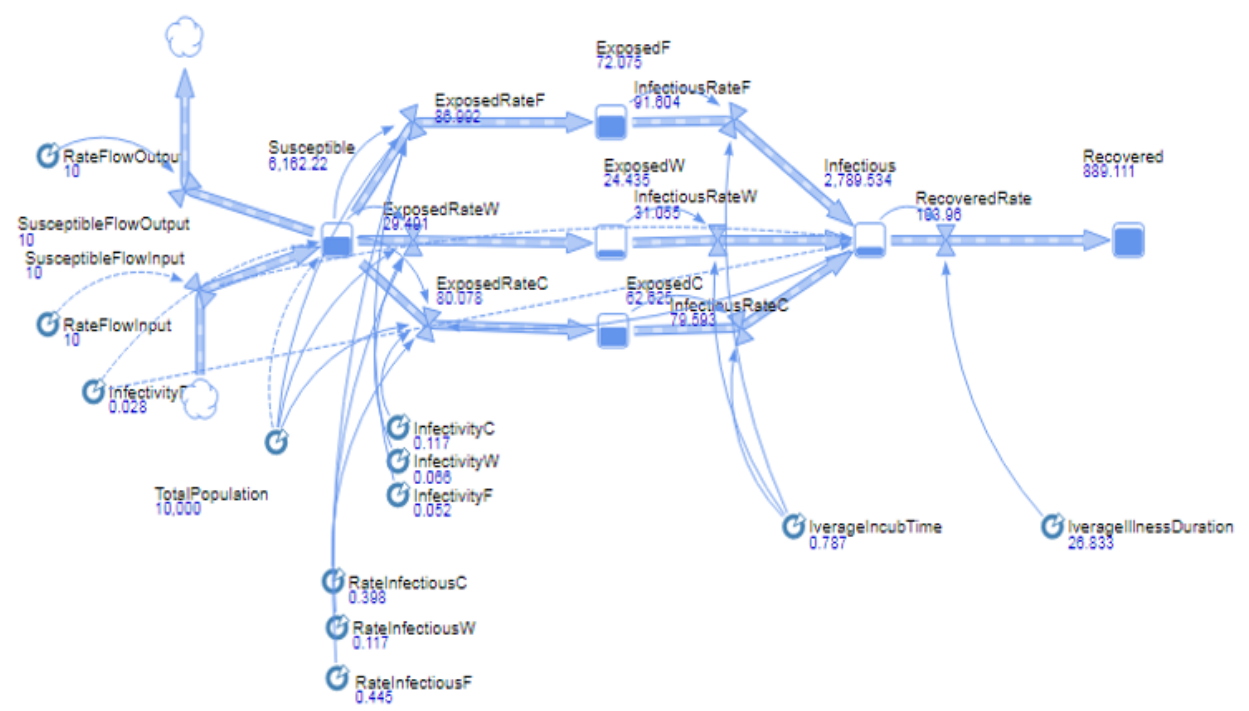

Fig. 1. Diagram of levels and flows.

Mathematically, a system-dynamic model is a system of differential equations that are solving numerically when the model is running. In our case, the functioning of the model is described by the following system of differential equations (the description of the variables is given in Table 1):

$$
\frac{d S}{d t}=r_{S N}-r_{E C}-r_{E W}-r_{E F}-r_{S O}
$$




$$
\begin{gathered}
\frac{d E_{C}}{d t}=r_{E D}+r_{E C}-r_{I C} \\
\frac{d E_{W}}{d t}=r_{E W}-r_{I W} \\
\frac{d E_{F}}{d t}=r_{E F}-r_{I F} \\
\frac{d I}{d t}=r_{I C}+r_{I W}+r_{I F}-r_{R} \\
\frac{d R}{d t}=r_{R} \\
S(0)=N-r_{S D} p_{D} \quad I(0)=r_{S D} p_{D} \\
E_{C}(0)=E_{W}(0)=E_{F}(0)=R(0)=0
\end{gathered}
$$

Here

$$
\begin{aligned}
& r_{S N}=i_{N} \quad r_{S O}=i_{O} \\
& r_{E C}=\frac{S}{N} \cdot I \cdot i_{C} \cdot p_{C} \quad r_{E W}=\frac{S^{2}}{N} \cdot i_{W} \cdot p_{W} \quad r_{E F}=\frac{S^{2}}{N} \cdot i_{F} \cdot p_{F} \\
& r_{I C}=\frac{E_{C}}{t_{i n}} \quad r_{I W}=\frac{E_{W}}{t_{i n}} \quad r_{I F}=\frac{E_{F}}{t_{i n}} \quad r_{I W}=\frac{I}{t_{i l}} .
\end{aligned}
$$

Several types of system dynamics models were considered (the model parameters are fixed, random, set using control elements).

Figure 2 shows the simulation results when parameters changes by using controls. The model makes it possible to trace the dynamics of the epidemic spread.

Table 1. Parameters and variables of the system dynamics model.

\begin{tabular}{|c|c|c|}
\hline Name & $\begin{array}{c}\text { Designation on flow } \\
\text { diagram }\end{array}$ & $\begin{array}{c}\text { Brief } \\
\text { designation }\end{array}$ \\
\hline \multicolumn{2}{|c|}{ Parameters } & \multicolumn{2}{|c|}{} \\
\hline Total Population & TotalPopulation & $\mathrm{N}$ \\
\hline $\begin{array}{c}\text { Contact } \\
\text { Water } \\
\text { food }\end{array}$ & $\begin{array}{c}\text { Infectivity } \\
\text { Infectivity }\end{array}$ & $\begin{array}{c}\mathrm{p}_{\mathrm{C}} \\
\mathrm{p}_{\mathrm{W}} \\
\mathrm{p}_{\mathrm{F}}\end{array}$ \\
\hline Infectivity & $\mathrm{p}_{\mathrm{D}}$ \\
\hline Proportion of infected visitors (deliveries) & & $\mathrm{i}_{\mathrm{C}}$ \\
\hline Intensity of infection & RateInfectiousC & $\mathrm{i}_{\mathrm{W}}$ \\
contact & RateInfectiousW & $\mathrm{i}_{\mathrm{F}}$ \\
\hline Water & RateInfectiousF & $\mathrm{i}_{\mathrm{N}}$ \\
\hline food & RateFlowInput & $\mathrm{io}_{\mathrm{O}}$ \\
\hline The duration of the incubation (latent) period & RateFlowOutput & $\mathrm{t}_{\mathrm{in}}$ \\
\hline Duration of infection & IverageIncubTime & $\mathrm{t}_{\mathrm{i}}$ \\
\hline
\end{tabular}




\begin{tabular}{|c|c|c|}
\hline \multicolumn{3}{|l|}{ Levels } \\
\hline Number of susceptible & Susceptible & $\mathrm{S}$ \\
\hline $\begin{array}{c}\text { Number of infected } \\
\text { Contact } \\
\text { Water } \\
\text { Food } \\
\end{array}$ & $\begin{array}{c}\text { ExposedC } \\
\text { ExposedW } \\
\text { ExposedF }\end{array}$ & $\begin{array}{l}E_{C} \\
E_{W} \\
E_{F}\end{array}$ \\
\hline $\begin{array}{c}\text { The number of infected in the active phase of the } \\
\text { disease }\end{array}$ & $\begin{array}{l}\text { InfectiousC } \\
\text { InfectiousW } \\
\text { InfectiousF }\end{array}$ & $\begin{array}{l}\mathrm{I}_{\mathrm{C}} \\
\mathrm{I}_{\mathrm{W}} \\
\mathrm{I}_{\mathrm{F}}\end{array}$ \\
\hline Number of recovered & Recovered & $\mathrm{R}$ \\
\hline \multicolumn{3}{|l|}{ Flows } \\
\hline Visitor flow rate & SusceptibleFlowInput & rSN \\
\hline Flow rate of leaving & SusceptibleFlowOutput & rso \\
\hline $\begin{array}{c}\text { Infestations flow rate } \\
\text { Contact } \\
\text { Water } \\
\text { Food }\end{array}$ & $\begin{array}{c}\text { ExposedRateC } \\
\text { ExposedRateW } \\
\text { ExposedRateF }\end{array}$ & $\begin{array}{l}\mathrm{r}_{\mathrm{EC}} \\
\mathrm{r}_{\mathrm{EW}} \\
\mathrm{r}_{\mathrm{EF}}\end{array}$ \\
\hline $\begin{array}{c}\text { Infection flow rate } \\
\text { Contact } \\
\text { Water } \\
\text { Food } \\
\end{array}$ & $\begin{array}{l}\text { InfectiousRateC } \\
\text { InfectiousRateW } \\
\text { InfectiousRateF }\end{array}$ & $\begin{array}{l}\mathrm{r}_{\mathrm{IC}} \\
\mathrm{r}_{\mathrm{IW}} \\
\mathrm{rIF}\end{array}$ \\
\hline Recovery flow rate & RecoveredRate & $\mathrm{r}_{\mathrm{R}}$ \\
\hline
\end{tabular}
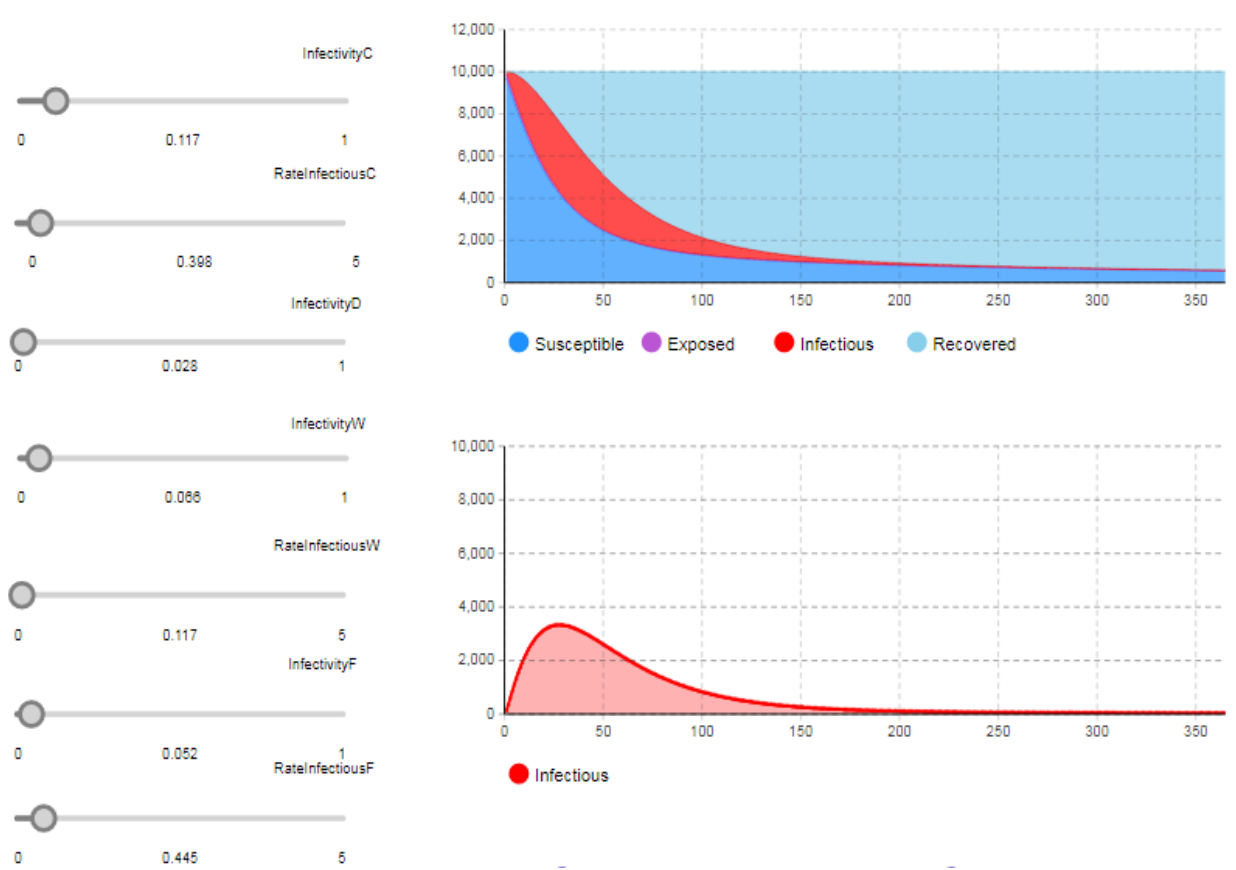

Fig. 2. The dynamics of epidemiological process, obtained using the system dynamics model.

\section{Stochastic agent-based model of epidemic spread}


There were considered several types of agent-based models (model parameters are fixed, random, set using control elements, various options for transitions between blocks).

Three state diagrams determine Individual behavior: a health state diagram, a location diagram, and a vaccination diagram (Figure 3). The description of the parameters and variables used in the diagrams is given in Table 2.

Table 2. Parameters and variables of the agent-based model.

\begin{tabular}{|l|c|}
\hline \multicolumn{1}{|c|}{ Name } & Designation \\
\hline Infection intensity & inf \\
\hline Waterborne contamination intensity & $\mathrm{iW}$ \\
\hline Food contamination intensity & $\mathrm{iF}$ \\
\hline Contact contamination intensity (trip contamination) & $\mathrm{iT}$ \\
\hline Contact intensity (trip) & $\mathrm{iGoTr}$ \\
\hline Vaccination intensity & $\mathrm{iVac}$ \\
\hline The probability of waterborne contamination & $\mathrm{pW}$ \\
\hline The probability of food contamination & $\mathrm{pF}$ \\
\hline The probability of contact contamination & $\mathrm{pT}$ \\
\hline The probability of taking action to vaccinate & $\mathrm{pVac}$ \\
\hline The probability of getting vaccinated & $\mathrm{pGoVac}$ \\
\hline The probability of contact (trip) & $\mathrm{pGoTr}$ \\
\hline Duration of the incubation period & $\mathrm{tInc}$ \\
\hline Duration of the disease & $\mathrm{tIll}$ \\
\hline Trip duration & $\mathrm{tTr}$ \\
\hline Start vaccination time & $\mathrm{tVac}$ \\
\hline Maximal number of infected for trip refusal per 100 person & MaxNInfTr \\
\hline Minimal number of infected to start vaccination per 100 person & MinNInfVac \\
\hline Current daily infected number per 100 person & NInf1 \\
\hline Maximal daily contaminated number per 100 person & kMaxNInf \\
\hline
\end{tabular}

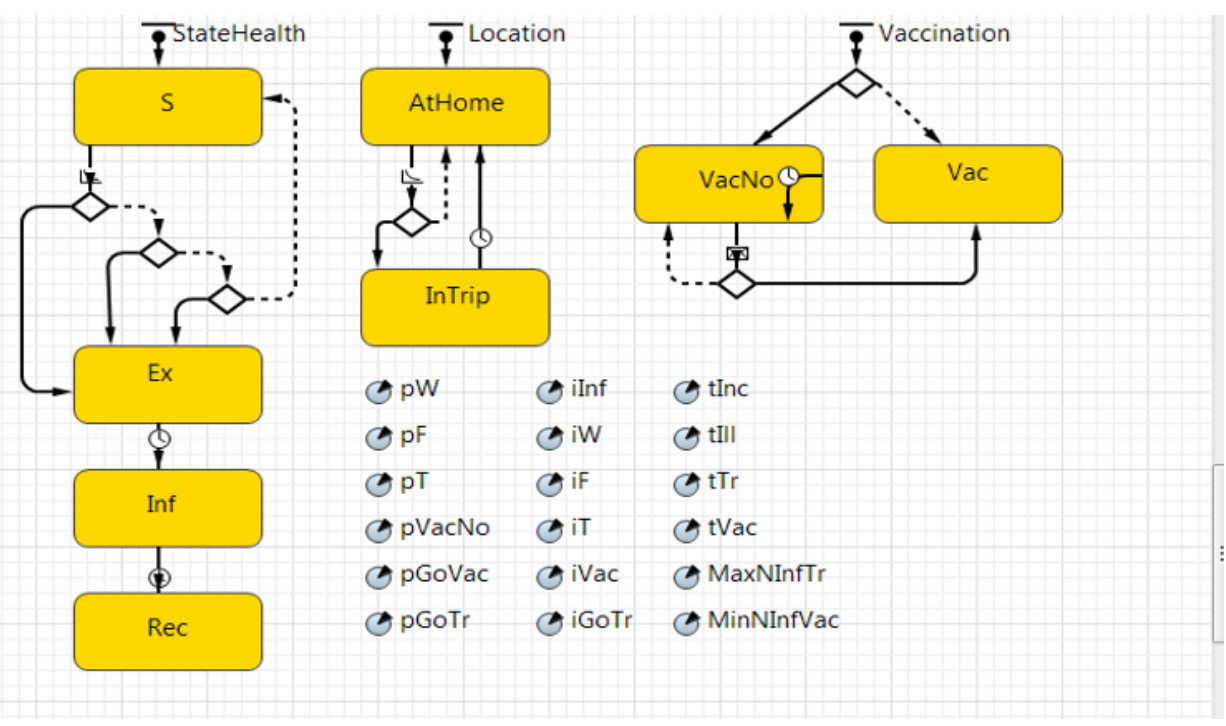

Fig. 3. Statecharts of the agent-based model.

The Vaccination diagram specifies the states VacNo "not vaccinated" and Vac "vaccinated". It is believed that since the onset of the epidemic, there are already a certain 
number of vaccinated people, determined by the $\mathrm{pVacNo}$ parameter (proportion of those not vaccinated). The Vaccination diagram uses internal and external transitions. The internal transition is triggered by a timeout with the $\mathrm{tVac}$ parameter, which determines the time after which the vaccination activities begin. It includes the sending of notifications inviting everyone who has not yet been ill to vaccinate, and the vaccination. The external transition between the states VacNo and Vac is carried out with a given probability pGoVac with additional restrictions taking into account the number of cases at the current moment and the current states of the agent in the StateHealth and Locaition diagrams.

The simulation results are displayed on time charts and diagrams (Figure 4). Here's the change of

a) the number of infected over time;

b) the ratio between the number of person in the four groups (susceptible, latent, infected and recovered);

c) the ratio between the number of person staying at home and in the trip;

d) the ratio between unvaccinated and vaccinated.

a)
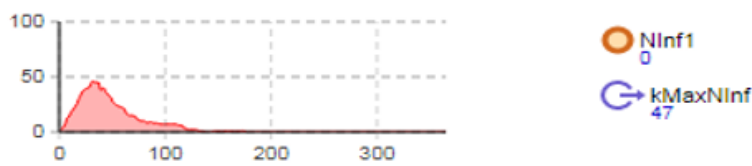

b)

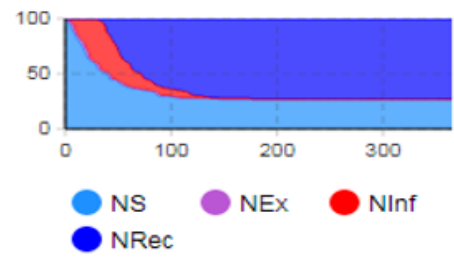

c)
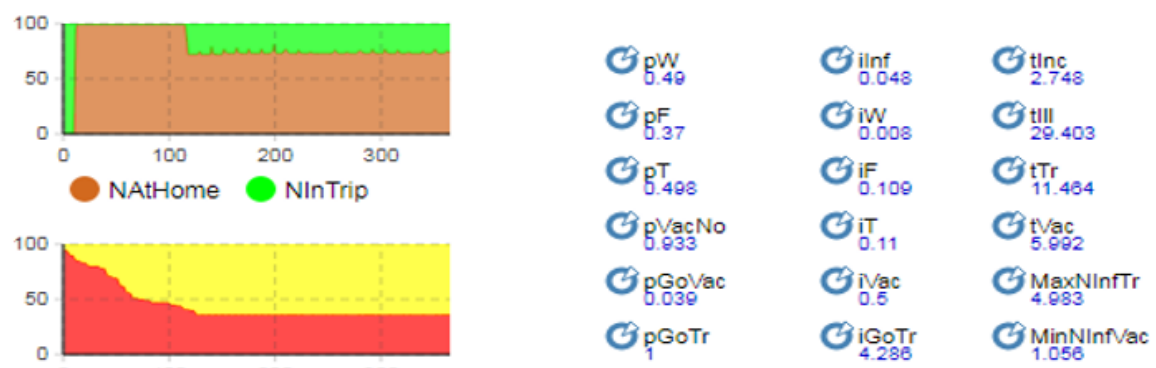

d)

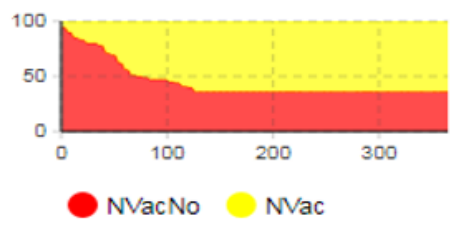

Fig. 4. Timelines and charts of the agent model.

One can use these agent-based models to predict the time of the disease peak, to determine the index of contagiousness depending on the external model parameters, to determine events (timing, intensity, etc.) to prevent the infection growth. 


\section{Conclusion}

The combination of these 2 models gives more possibilities for their application in a real situation. A system-dynamic model designed for strategic modeling of the epidemiological situation, reflecting global trends, in combination with an agent-based model, will also allow using tactical nuances. This will make it possible to influence the situation, to reduce the epidemic growth rate and reduce the peak of the disease.

The agent-based approach in combination with the system-dynamic model seems to be the most promising, since it is more realistic. It allows describing the individual behavior of each agent - person, who independently form events conditioning transitions between states.

\section{References}

1. Kondrat'ev M A 2013 Computer research and modeling 5(5) 863-882

2. Efimova N V, Gornov A Yu, Zarodnyuk T S 2010 Human Ecology 3 3-7

3. Moskvitina E A, Yanovich E G, Kurylenko M L, et al 2020 Forecast for 2020 Problems of especially dangerous infections 2 38-47

4. Moskvitina E A, Tyuleneva E G, Kruglikov V D, et al 2018 Forecast for 2020 Problems of especially dangerous infections 1 36-43

5. Bashabsheh M M 2013 Naukovedenie Internet journal 6 127-135

6. Kermack W O, McKendrick A G 1927 Proc R Soc Lond A 115 700-721 